\title{
CAN STUDENT AND STAFF INVOLVEMENT IN QUALITY ASSURANCE AND PROMOTION BE ACHIEVED IN THE HEALTH SCIENCES THROUGH mHEALTH AND e-LEARNING? A CONCEPTUAL PLATFORM DESIGN
}

\author{
H. Noorbhai \\ Department of Sport and Movement Studies \\ University of Johannesburg \\ Johannesburg, South Africa \\ e-mail: habibn@ui.ac.za / https://orcid.org/0000-0001-9464-6854
}

\section{ABSTRACT}

With the new generation of health professionals, some skills such as diagnosing and treating an array of diseases has become complex and challenging. These health professionals also seem to portray other transferrable skills which can be embedded with future curricular through the use of e-learning. It has been reported that universities have a responsibility and an opportunity to effectively prepare future health professionals to address health related issues with evolving changes within the healthcare landscapes through enhanced student involvement. Practical activities based on local institutional creativity and regional issues can reinvigorate the links between clinical practice and the health of people, through quality assurance and promotion. One form of a practical activity that can be identified is through the use of e-learning and technology. Over the last decade, developments in e-learning and technologies are creating the groundwork for health sciences education. There may be a similar trajectory within the health sciences domain where both health sciences students and qualified health professionals frequently use their mobile phones when consulting with patients. Given the diversity of e-learning methods, there are many ways to carry out such an evaluation. However, the current literature shows us that we have yet to reach any form of consensus about which indicators to evaluate. There is a greater need for an evaluation tool or platform that is properly constructed, validated and tested within the health sciences, that can also be used to enhance student involvement and quality assurance. Given the limited success rate of e-learning within the health sciences field, this article aims to fill this gap by proposing how such an mHealth and e-learning methodology for a platform can be evaluated and established, to optimise learning (for students and academic staff), student involvement, quality assurance and healthcare (for patients) in South Africa.

Keywords: mHealth, e-learning, quality assurance, promotion, health sciences

\section{INTRODUCTION}

South Africa has a quadruple burden of disease from human immunodeficiency virus/acquired immunodeficiency syndrome and tuberculosis, diabetes, maternal and child mortality, injuries 
and violence, and non-communicable diseases (NCDs; National Department of Health 2013; Bradshaw, Groenewald and Laubscher 2003; Mayosi et al. 2012; Stats SA 2014; Pillay-van Wyk, Msemsuri and Laubscher 2016). Despite the epidemic and epidemiology of these diseases globally and in South Africa, it has been postulated that if the practice of medicine is to be more than identifying and treating disease that includes a significant preventative component, then there is a need to better understand the relationships between ecosystem health and human health (Rapport et al. 2003). However, health science faculties and medical schools, particularly in Low and Middle-Income Countries (LMIC) make limited provision for teaching innovative healthcare approaches when addressing burden of diseases (Lozano, Naghavi and Foreman 2012), preventive medicine and treatment, due to the already overloaded curricula. There is, therefore, a need to find innovative solutions to supporting current and future health professionals in correctly diagnosing, treating and preventing diseases from an array of diseases. Among high-income countries, topics on health sciences education in varied faculties had embraced the challenge of widening their conventional curriculums. However, among LMIC, this has not been the case, and, as such, there is a need to fully expand the curriculum and challenge in these countries.

\section{e-Learning in the Health Sciences}

Epistemology, ontology and axiology have laid the foundations for how we, as individuals, understand the world we live in, the determinations we make about issues relating to truth, and the matters we consider to be of value to us individually, and to society at large (Edelheim 2014). In line with these foundations, we have begun to learn the five trends shaping the future of work that include: new behaviours, new technologies, the millennial workforce, mobility and globalisation (Morgan 2014). People are able to access knowledge anywhere, anytime and virtually. In the context of health sciences education, we have witnessed that such curricula has changed, in order to meet the evolving demands of the healthcare system.

One such change and evolvement in the curricula is e-learning, which is also known as web-based learning, online learning, distributed learning, computer-assisted instruction, or internet-based learning. Historically, there have been two common e-learning modes: distance learning and computer-assisted instruction. Distance learning uses information technologies to deliver instruction to learners who are at remote locations from a central site (Ruiz, Mintzer and Leipzig 2006). Computer-assisted instruction (also called computer-based learning and computer-based training) uses computers to aid in the delivery of stand-alone multimedia packages for learning and teaching (Ward et al. 2001). These two modes are categorised under e-learning because over the last two decades, the internet has become the integrating technology 
for many forms and modes of learning platforms (Ruiz et al. 2006).

Almost 15 years ago, Ruiz et al., (2006) indicated that the integration of e-learning into undergraduate, graduate, and continuing health sciences education will promote a shift towards adult learning in health sciences education. This is where educators will no longer serve solely as distributors of content, but become facilitators of learning and assessors of competency. It is now an established fact that evaluating the effects of e-learning design can be complicated.

With the new generation of health professionals, some skills such as diagnosing and treating an array of diseases has become complex and challenging (Frenk et al. 2010). These health professionals also seem to portray other transferrable skills which can be embedded with future curricular through the use of e-learning. It has been reported that universities have a responsibility and an opportunity to effectively prepare future health professionals to address health related issues with evolving changes within the healthcare landscapes through enhanced student involvement (Jogerst et al. 2015). Practical activities based on local institutional creativity and regional issues can reinvigorate the links between clinical practice and the health of people, through quality assurance and promotion. One form of a practical activity that can be identified is through the use of e-learning and technology (Walpole et al. 2019).

\section{mHealth to fill curricula gaps}

Over the last decade, developments in e-learning and technologies are creating the groundwork for a revolution in education (Masic 2008). This may be a similar trajectory within health sciences education where both training doctors and qualified doctors frequently use their mobile phones when consulting with patients. This is also known as tele-education, which is also observed through health education on distance, using Information Communication Technologies (ICTs), as well as continuous education of health system beneficiaries and the use of electronic libraries, databases or electronic data with databases of knowledge (Masic 2008). In the knowledge economy that we currently reside in, and more broadly, with the rapid evolvement of research within the public health sphere (Noorbhai 2013), it can become quite challenging to streamline important information such as symptoms, exposures, treatments and prevention strategies of a disease for health sciences students and health professionals. One could argue that mobile health (mHealth) could be a solution.

More developments of mHealth, digital applications and tele-medicine has emerged, which has either been used in healthcare settings or designed for health professionals (Mosa, Yoo and Sheets 2012; Ozdalga, Ozdalga and Ahuja 2012; Wallace, Clark and White 2012; Free et al. 2013; Payne, Weeks and Dunning 2014; O’Donovan, Bersin and O'Donovan 2015; Gagnon et al. 2016). Recently, a study conducted by De La Cruz, Monroy and Mosahebi (2019) 
showed that although there are further studies required to assess the impact that mobile applications have on patients' care and healthcare professional services, there is an overall positive impression of the use of perioperative mHealth applications. Yaghobian et al. (2019) elaborated that further research would need to be conducted on tele-medicine education, training implementation and knowledge, attitudes and practices of health sciences students, health professionals and resident medical doctors.

\section{Rationale for the platform}

Given the diversity of e-learning methods, there are many ways to carry out such an evaluation. However, the current literature shows us that we have yet to reach any form of consensus about which indicators to evaluate. There is a greater need for an evaluation tool or platform that is properly constructed, validated and tested within the health sciences, that can also be used to enhance student involvement and quality assurance. Given the limited success rate of e-learning within the health sciences field, this article aims to fill this gap by discussing how such an mHealth and e-learning platform can be evaluated and established, to optimise learning (for students and academic staff), student involvement, quality assurance and healthcare (for patients) in South Africa.

mHealth technologies also have the potential to greatly impact health research, healthcare, health outcomes, and learning. However, it has also been shown that the exponential growth of the technology has outpaced the science of mHealth (Nilsen et al. 2012). Due to some health sciences curricula having no room to include mHealth training, and practicing professionals do not have the time to research these approaches or mediums, further research would fill this gap and warrants the need to better understand students and the healthcare setting in the fourth industrial revolution (4IR). It also warrants the need to develop an application and platform, as well as a strong network, that will bridge this gap (between mHealth and e-learning in healthcare technology and innovation) in the health sciences fraternity. Given the unsuccessful rate of e-learning within the health sciences area (Lindenmaier et al. 2018), a virtual centre (focusing on healthcare innovation) and research project has been conceptualised to fill this void by researching how such a platform and curricula can be merged and subsequently evaluated and established, to optimise both learning and healthcare with patients, as well as quality assurance and student involvement for health sciences students.

\section{METHODOLOGY}

A mixed-methods research study should be conducted prior to the development of the platform and curricula (Figure 1). There is currently no consensus on an optimal platform and evaluation 
tool that can be utilised in designing a curriculum for effective dissemination for e-learning that can be used by health sciences students, through health technology teaching (Figure 2).

Note: Figure 2 is an extension of the teaching and learning arm from Figure 1.



Figure 1: Three core pillars for digital health adoption: A preliminary model



Figure 2: Overview for effective health technology teaching and quality assurance

\section{Research design and setting}

A mixed-methods study combining both qualitative and quantitative analysis is found to be more effective and invaluable for gaining insights from participants. Therefore, this study will employ a mixed-methods study design in which a survey will be utilised including both closedended and open-ended questions, as well as focus group discussions that will be conducted before and after the surveys takes place. 


\section{Study procedure and study participants}

Focus Group Discussions (FGDs) $(\mathrm{N}=50-100)$ will be conducted among both health science students (medicine, physiotherapy, biokinetics, optometry, nursing, occupational therapy, dentistry and other allied health or medical students) and staff from five universities that are regarded among the top Health Sciences Faculties in South Africa according to Times Higher Education rankings (University of Cape Town, University of the Witwatersrand, University of Johannesburg, University of Pretoria and the University of Stellenbosch) in South Africa. A survey will also be sent out to all health science students at the universities $(n=500)$. A probability calculation indicates that 500 would be a sufficient number of study subjects. Students and universities will be contacted through internal networks as well as through the Faculty Administration Offices at the universities.

\section{Survey questionnaire}

After validation and standardisation of the survey, an online survey will be developed using GoogleForms ${ }^{\mathrm{TM}}$ which will be provided to health science students and staff. Each survey may require between 15-20 minutes for its completion. The questions asked will be both open and closed-ended questions. The online survey will also be sent to three experts in the field to have it validated, verified and piloted among a few students, prior to releasing it in order to ensure validation. Any changes suggested will be made to the survey.

\section{Focus group discussions}

The purpose of the focus group discussion (FGDs) would be to refine the survey questionnaire, recruit participants for an online survey, review the findings from the FGDs and be in a space to make further recommendations. The variety of the respondents we intend engaging with can influence the contribution our study is able to make towards the development of the survey questionnaire. As such, participants will be intentionally selected using a sampling technique described as "purposeful sampling". The duration of the FGDs will range between 20 to 35 minutes and guide questions will be developed for the discussions. These questions aim to solicit the respondents' perceptions with regards to mHealth and e-learning within the health sciences curricula. The responses from both focus group discussions and the surveys would allow and ensure optimal development for the mHealth and e-learning platform/application.

\section{Quantitative data analysis}

Data will be captured automatically from GoogleSheets ${ }^{\mathrm{TM}}$ and exported onto an online Microsoft Excel sheet upon submission of the students' responses. Pearson's correlations and chi-squared tests will be conducted to determine any significant trends and relationships in the 
answers obtained from the survey. All statistical analyses will be performed using SPSS (Version 26, IBM) at a significance level of $p=0.05$.

\section{Qualitative data analysis}

Atlast.ti will used for analysis of the open-ended data of the survey as well as for the two focus groups. Once the questionnaire is answered and after the focus group discussions are conducted, open-ended answers and transcriptions gathered from the students will be coded using a thematic coding framework. These codes will be formulated from the answers provided by the students. The results will be discussed as themes that arise from the quantitative data, which will be supported by qualitative data in the form of quotes and insights provided by the students.

\section{Culture and considerations}

Many health sciences faculties within universities in South Africa do not foster a dynamic culture of e-learning and mHealth within their health sciences curriculums. Such a culture would also assist in creating more opportunities within the healthcare landscapes for job creation. Some universities in South Africa are embedding the ethos of 4IR (such as the University of Johannesburg), but are yet to inculcate the use of e-learning within their health sciences curricula (and many of their other faculty curricula). Consistent engagement in the conception or creation of the platform whereby it will be used for health sciences curricula and e-learning benefits within the mHealth domain as well assisting health professionals when consulting patients, is lacking. One has to also take into account some of the considerations and limitations that exist when streamlining quality assurance of digital health in South Africa:

- $\quad$ Strength and infrastructure of internet and Wi-Fi capability at universities.

- Student projects as part of their hours/final-year degree.

- Longevity and sustainability of the use of a platform, device or project.

- Data costs and challenges in rural areas.

- $\quad$ Policies, governance and legalities involved need to be streamlined.

- $\quad$ Diverse languages.

- Designing for a wider group followed by replication in rural areas for accessibility.

- A review is needed on what devices are used in rural areas, as well as whether Android or Apple are more common for utilisation.

Furthermore, according to the WHO (2013), a report discussing the transformation and scale up of health professionals' education and training mentioned the following: "scaling up 
education and training is a critical component of the strategies to strengthen the health workforce, but much of its effectiveness will be lost if it is not complemented with policies to retain the graduates, and to provide them with working conditions that will enable them to use their knowledge and skills productively". This is imperative to consider in the research methodology and development of the platform. The platform would also need to consider to foster the use of effective strategies of promotion, disease prevention, and health sciences education.

\section{CONCLUSION}

Change is inevitable. Education has evolved and adapted through the digital and electronic ages. It is only a matter of time before teaching and learning adopts changes through the technological age. Now is as desirable a time as any to streamline and adapt the health sciences curricula for the promotion of health sciences education in the current 4IR through a platform.

\section{REFERENCES}

Bradshaw, D., P. Groenewald and R. Laubscher. 2003. Initial burden of disease estimates for South Africa, 2000. South African Medical Journal 93: 682-688.

De La Cruz Monroy, M. F. I. and A. Mosahebi. 2019. The use of smartphone applications (Apps) for enhancing communication with surgical patients: A systematic review of the literature. Surgical Innovation 2. doi: 10.1177/1553350618819517.

Edelheim, J. R. 2014. Ontological, epistemological and axiological issues. In The Routledge handbook of tourism and hospitality education, 62-74. Routledge.

Free, C., G. Phillips, L. Watson, L. Galli, L. Felix, P. Edwards, V. Patel and A. Haines. 2013. The effectiveness of mobile-health technologies to improve health care service delivery processes: A systematic review and meta-analysis. PLoS Medicine 10: e1001363. doi:10.1371/ journal.pmed.1001363.

Frenk, J., L. Chen, Z. A. Bhutta, J. Cohen, N. Crisp, T. Evans, H. Fineberg, P. Garcia, Y. Ke, P. Kelley and B. Kistnasamy. 2010. Health professionals for a new century: Transforming education to strengthen health systems in an interdependent world. The Lancet 376(9756): 1923-1958.

Gagnon, M. P., P. Ngangue, J. Payne-Gagnon and M. Desmartis. 2016. m-Health adoption by healthcare professionals: A systematic review. Journal of the American Medical Informatics Association 23: 212-220.

Jogerst, K., B. Callender, V. Adams, J. Evert, E. Fields, T. Hall, J. Olsen, V. Rowthorn, S. Rudy, J. Shen and L. Simon. 2015. Identifying interprofessional global health competencies for 21st-century health professionals. Annals of Global Health 81(2): 239-247.

Lindenmaier, T. J., J. Brown, L. Ranieri, D. Steary, H. Harrison, J. Flook and E. Lorusso. 2018. The effect of an e-learning module on health sciences students' venipuncture skill development. Canadian Journal of Respiratory Therapy: CJRT= Revue canadienne de la therapie respiratoire: RCTR 54(1): 12.

Lozano, R., M. Naghavi and K. Foreman. 2012. Global and regional mortality from 235 causes of death for 20 age groups in 1990 and 2010: A systematic analysis for the global burden of disease study 2010. The Lancet 380: 2095-2128.

Masic, I. 2008. E-learning as new method of medical education. Acta Informatica Medica 16(2): 102117. doi: 10.5455/aim.2008.16.102-117. 
Mayosi, B. M., J. E. Lawn, A. van Niekerk, D. Bradshaw, S. S. Abdool Karim, H. M. Coovadia and Lancet South Africa team. 2012. Lancet South Africa team. Health in South Africa: Changes and challenges since 2009. The Lancet 380: 2029-2043.

Morgan, J. 2014. The future of work: Attract new talent, build better leaders, and create a competitive organization. John Wiley \& Sons.

Mosa, A. S., I. Yoo and L. Sheets. 2012. A systematic review of healthcare applications for smartphones. BMC Medical Informatics and Decision Making 12: 67. doi: 10.1186/1472-6947-12-67.

National Department of Health. 2013. Strategic plan for the prevention and control of noncommunicable disease 2013-17. https://www.health-e.org.za/wpcontent/uploads/2013/09/NCDs-

Nilsen, W., S. Kumar, A. Shar, C. Varoquiers, T. Wiley, W. T. Riley, M. Pavel and A. A. Atienza. 2012. Advancing the science of mHealth. Journal of Health Communication 17(Sup1): 5-10.

Noorbhai, M. H. 2013. A public health approach to increase physical activity and health education: The Biokinetic Humanitarian Project. African Journal for Physical Health Education, Recreation and Dance 19(4_2): 993-998.

O'Donovan, J., A. Bersin and C. O'Donovan. 2015. The effectiveness of mobile health (mHealth) technologies to train healthcare professionals in developing countries: A review of the literature. BMJ Innovations 1: 33-36.

Ozdalga, E., A. Ozdalga and N. Ahuja. 2012. The smartphone in medicine: A review of current and potential use among physicians and students. Journal of Medical Internet Research 14: e128.

Payne, K. F., L. Weeks and P. Dunning. 2014. A mixed methods pilot study to investigate the impact of a hospital-specific iPhone application (iTreat) within a British junior doctor cohort. Health Informatics Journal 20: 59-73.

Pillay-van Wyk, V., W. Msemburi, R. Laubscher, R. E. Dorrington, P. Groenewald, T. Glass, B. Nojilana, J. D. Joubert, R. Matzopoulos, M. Prinsloo and N. Nannan. 2016. Mortality trends and differentials in South Africa from 1997 to 2012: Second National Burden of Disease Study. The Lancet Global Health 4(9): e642-e653.

Rapport, D. J., J. Howard, R. Lannigan and W. McCauley. 2003. Linking health and ecology in the medical curriculum. Environment International 29(2-3): 353-358.

Ruiz, J. G., M. J. Mintzer and R. M. Leipzig. 2006. The impact of e-learning in medical education. Academic Medicine 81(3): 207-212.

Stats SA see Statistics South Africa.

Statistics South Africa. 2014. Mortality and causes of death in South Africa, 2012: Findings from death notification/Statistics South Africa. Statistical release P0309.3. Pretoria: Statistics South Africa.

Wallace, S., M. Clark and J. White. 2012. "It's on my iPhone": Attitudes to the use of mobile computing devices in medical education, a mixed-methods study. BMJ Open 2. doi: 10.1136/bmjopen-2012001099 .

Walpole, S. C., S. Barna, J. Richardson and H. A. Rother. 2019. Sustainable healthcare education: Integrating planetary health into clinical education. Doctopic: Analysis and Interpretation. S25425196(18)30246-8

Ward, J. P., J. Gordon, M. J. Field and H. P. Lehmann. 2001. Communication and information technology in medical education. The Lancet 357(9258): 792-796.

WHO see World Health Organization.

World Health Organization. 2013. Transforming and scaling up health professionals' education and training: World Health Organization guidelines 2013. World Health Organization.

Yaghobian, S., R. Ohannessian, A. Mathieu-Fritz and T. Moulin. 2020. National survey of telemedicine education and training in medical schools in France. Journal of Telemedicine and Telecare 26(5): 303-308. doi: 10.1177/1357633X18820374. 